\title{
PRINCIPIOS IMPLÍCITOS Y FUENTES SOCIALES DEL DERECHO*
}

\author{
Juan Pablo Alonso ** \\ Universidad de Buenos Aires \\ jalonso@derecho.uba.ar
}

RESUMEN. En este trabajo desarrollaré algunas ideas tendentes a elucidar el modo en que los juristas justifican la validez de principios implícitos. Sintetizando, intentaré responder a preguntas del tipo ¿cuál es la explicación lógica o teórica respecto del modo en que los juristas obtienen principios implícitos? o, ¿cuándo un enunciado normativo vale como un principio implícito de un sistema jurídico determinado?

Intentaré demostrar que algunas críticas de Ronald DwORKIN contra el positivismo no son adecuadas, en punto a que es posible justificar la vigencia (existencia, validez, etc.) de principios implícitos con arreglo a la tesis juspositivista de las fuentes sociales del derecho y con prescindencia de fuentes morales. rencia.

Palabras clave: principios implícitos, fuentes sociales del derecho, abducción, cohe-

\section{Implicit Principles and Social Sources of Law}

ABSTRACT. In this paper I will develop some ideas to elucidate the way jurists justify the validity of implicit principles. Summarizing, I will try to answer questions such as: what is the logical or theoretical explanation of how jurists obtain implicit principles? Or, when is a normative statement considered an implicit principle of a given legal system?

I will try to show that some criticisms of Ronald DwORKIN against positivism are not adequate, in that it is possible to justify the validity (existence, etc.) of implicit principles according to the juspositivist thesis of the social sources of law and without reference to moral sources.

Keywords: implicit principles, social sources of law, abduction, coherence.

* Fecha de recepción: 12 de junio de 2017. Fecha de aceptación: 18 de octubre de 2017.

** El presente trabajo es fruto de un proyecto de investigación que dirigí junto a Gabriela SCATAGLINI, con financiación de la Facultad de Derecho de la Universidad de Buenos Aires, bienio 2014/2016. DECyT 1403 «Principios Jurídicos Implícitos». 


\section{LOS PRINCIPIOS IMPLÍCITOS EN DWORKIN Y SUS CRÍTICAS AL JUSPOSITIVISMO}

\subsection{Algunas críticas de DwORKIN a HART}

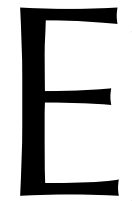

n varios de sus trabajos Ronald DwORKIN ha criticado al juspositivismo, especialmente la versión teórica desarrollada por Herbert HART. Según DwORKIN, el juspositivismo es un modelo «de y para las reglas» incapaz de dar cuenta de estándares normativos diferentes de las reglas como lo son los principios jurídicos implícitos del tipo «nadie se puede beneficiar de su propio accionar ilícito».

DWORKIN efectúa numerosas consideraciones sobre los principios jurídicos implícitos. En esta instancia destacaré solo dos de ellas: a) que la regla de reconocimiento de HART no sirve para identificar principios jurídicos implícitos, y $b$ ) que no es posible construir un test preciso que permita elucidar cuándo un enunciado normativo es un principio implícito de un determinado sistema jurídico; entre otras razones, porque la distinción hartiana entre creación y aplicación de normas de reglas no es trasladable a los principios implícitos.

De acuerdo con HART, la mayoría de las normas de derecho son válidas porque alguna institución competente las promulgó. Algunas fueron creadas por un órgano legislativo en forma de leyes. Otras fueron creadas por los jueces que las formularon para decidir casos particulares y las establecieron así como precedentes para el futuro. Pero este tipo de certificación no sirve para los principios de los casos Riggs y Henningsen, cuyo origen como principios jurídicos no se basa en una decisión particular de ningún tribunal u órgano legislativo, sino en un sentido de conveniencia y oportunidad que, tanto en el foro como en la sociedad, se desarrolla con el tiempo ${ }^{1}$.

No podríamos idear ninguna fórmula que sirviera para probar cuánto apoyo institucional, y de qué clase, es necesario para convertir un principio en principio jurídico².

Si estamos defendiendo el principio de que un hombre no puede beneficiarse de su propio delito, podríamos citar actas de tribunales y leyes que lo ejemplifiquen, pero esto se refiere tanto a la aceptación del principio como a su validez ${ }^{3}$.

\subsection{En defensa del juspositivismo. Los escenarios teóricos posibles}

A lo largo del presente trabajo intentaré refutar las reseñadas tesis de DwORKIN. Señalaré que es posible construir criterios tendentes a explicar cómo los jueces y los juristas obtienen o justifican principios implícitos como parte del derecho positivo sin recurrir a criterios sustantivos o de contenido. Señalaré, también, que la justificación de los principios implícitos no implica necesariamente traspasar los límites del positivismo, o en particular alguna de sus dos tesis principales (la tesis de las fuentes sociales y la tesis de la separación entre derecho y moral). Si esto es así, entonces no es con-

1 Dworkin, Los Derechos en serio, 1977, Barcelona, Ariel, 1984 (traducción al castellano de Marta GUASTAVINO), 94-95.

2 Ibid., 95

3 Ibid., 96 
ceptualmente necesario adoptar una postura antipositivista a la DWORKIN para poder participar en la discusión acerca de los principios implícitos de los sistemas jurídicos.

En definitiva, defenderé dos tesis en contra de DwORKIN: 1) es posible construir criterios metodológicos para justificar la vigencia de principios implícitos, y 2) es posible hacerlo dentro de los límites del derecho positivo.

Estas dos tesis muestran que, en materia de principios jurídicos implícitos, el escenario teórico es bastante más complejo que el modo en que lo presenta DwORKIN. En efecto, mientras que la posición de DwORKIN supone el rechazo mis tesis 1) y 2), podría haber posiciones no positivistas que acepten mi tesis 1 ) y rechacen mi tesis 2), por ejemplo, un jusnaturalista bien podría, a diferencia de DwORKIN, confiar en un «test moral» preciso, o fijar condiciones de adecuación a una fórmula con contenido moral $^{4}$; a su vez, dentro del juspositivismo también existiría espacio conceptual para ser escéptico (por motivos similares a los de DwORKIN) acerca de la posibilidad de construir una fórmula precisa o test de adecuación que sirva para justificar la vigencia de principios jurídicos implícitos 5 .

\section{TRES FORMAS DE JUSTIFICAR LA VIGENCIA DE PRINCIPIOS JURÍDICOS IMPLÍCITOS}

Siguiendo a Luis PRIETO SANCHÍs puede afirmarse que los principios jurídicos implícitos son enunciados normativos que no se extraen (no son el significado) de alguna disposición jurídica determinada ${ }^{6}$. Los juristas y dogmáticos suelen presentar al menos tres vías alternativas para explicar o justificar la vigencia (o existencia, o validez, etc.) de un principio implícito:

a) El principio implícito es una especificación (derivación, instanciación, etc.) de un principio jurídico explícito positivo más general.

b) El principio implícito es una generalización a partir de un conjunto de normas jurídicas positivas más específicas.

c) El principio tiene una justificación autónoma, con prescindencia de fuente normativa positiva alguna.

Las dos primeras vías de obtención de principios implícitos [vías a) y b)] invocan alguna fuente normativa positiva como punto de partida; la tercera vía $c$ ) no invoca fuente normativa positiva alguna.

En algunas oportunidades los juristas justifican la existencia de un determinado principio jurídico implícito por varias de estas tres vías a la vez; por ejemplo, sostienen que el principio implícito en cuestión es una generalización a partir de normas jurídicas válidas (más específicas que el principio implícito) y que, a la vez, el principio

${ }^{4}$ Esta es la posición de quienes defienden la existencia del «derecho natural» [por ejemplo, VIGO, La injusticia extrema no es derecho (De Radbruch a Alexy), Buenos Aires, La Ley, 2004].

5 Esta es la posición de Bulygin (BulYgin, 1966, «Sentencia judicial y creación de derecho», en ALCHOURRÓn y Bulygin, Análisis lógico y derecho, Madrid, Centro de Estudios Constitucionales, 1991, 355-369).

${ }^{6}$ Prieto Sanchís, Sobre principios y normas. Problemas del razonamiento jurídico, Madrid, Centro de Estudios Constitucionales, 1992, 141. 
implícito en cuestión es una instancia de un principio jurídico explícito más general (usualmente, algún principio constitucional).

PRIETO SANCHÍS señala que «un principio implícito es una norma que no puede ser considerada como el significado de determinada disposición normativa, sino que se obtiene a partir de una o varias disposiciones que se supone constituyen casos de aplicación o especificación de dicho principio» ${ }^{7}$. Riccardo GUASTINI, por su parte, considera que «el grado de confirmación del principio [implícito] es superior cuanto mayor sea el número de normas que pueden citarse como "casos" en los que el principio está presente» ${ }^{8}$. Manuel ATIENZA y Juan RUIZ MANERO, finalmente, distinguen entre principios explícitos y principios implícitos; los primeros son «formulados expresamente en el ordenamiento jurídico», y los segundos son «extraídos a partir de enunciados presentes en el ordenamiento jurídico» ${ }^{9}$. Asimismo, los autores señalan que los principios implícitos son razones para la acción que no son perentorias (al igual que los principios explícitos), ni independientes de su contenido, «porque si deben formar parte del razonamiento de los órganos jurisdiccionales no es por virtud de su origen en fuente alguna, sino por cierta cualidad de su contenido. Dicha cualidad no es su justicia intrínseca, $[\ldots]$ ni son simplemente pautas que gozan de arraigo social, [...] sino su adecuación o coherencia en relación con las reglas o principios basados en fuentes» ${ }^{10}$.

Las opiniones de PRIETO SANCHís y GUASTINI, aquí transcritas, se refieren al segundo modo en que los juristas obtienen principios implícitos, esto es, a partir del contenido de normas explícitas que son más específicas que la generalización contenida en el principio implícito.

Las opiniones de ATIENZA y RUIZ MANERO, en cambio, se refieren indistintamente a los dos primeros modos en que los juristas obtienen principios implícitos, esto es, se refieren tanto a principios implícitos extraídos a partir de reglas explícitas como a los extraídos por derivación a partir de principios explícitos más generales.

Frederick SCHAUER sostiene que las reglas «atrincheran» el balance de razones subyacentes en función de generalizaciones probabilísticas. Señala que el hecho de que la gente que va a un restaurante a comer desea hacerlo con tranquilidad y sin molestias (razón subyacente), más el hecho de que los perros en un restaurante pueden ladrar, corretear, saltar sobre los clientes y comer restos de comida del piso (generalización probabilística), llevan al dueño del restaurante a colocar en la entrada un cartel que expresa lo siguiente: «Prohibido el ingreso de perros» (la regla) ${ }^{11}$.

Las razones subyacentes de las reglas de SCHAUER parecen hacer referencia al segundo modo referenciado, dado que son descriptas como generalizaciones probabilísticas de ciertas evidencias (las diferentes molestias ocasionadas por los perros).

7 PRIETO SANChís, op. cit., 1992, 141.

8 GuASTINI, Dalle fonti alle norme, Torino, Giappichelli, 1990, 122 y ss.

9 Atienza y Ruiz Manero, Las piezas del derecho. Teoría de los enunciados jurídicos, Madrid, Ariel Derecho, 1996. Los autores señalan que la distinción entre principios explícitos e implícitos es exhaustiva y excluyente.

10 AtienZa y Ruiz Manero, op. cit., 1996, 13.

11 SChauer, Playing by the Rules. A Philosophical Examination of Rule - Based Decision- Making un Law and un Life, Oxford, Oxford University Press, 1991. 


\section{LOS PRINCIPIOS IMPLÍCITOS COMO ESPECIFICACIÓN DE PRINCIPIOS EXPLÍCITOS POSITIVOS MÁS GENERALES}

\subsection{Un análisis de SANCINETTI}

Marcelo SANCINETTI ${ }^{12}$ analiza un problema del Código Penal argentino respecto de la regulación de las lesiones dolosas y las lesiones imprudentes (culposas). Tal código categoriza tres tipos de lesiones dolosas, asignándoles escalas penales crecientes: lesiones leves, de un mes a un año (art. 89); lesiones graves, de uno a seis años (art. 90); y lesiones gravísimas, de tres a diez años (art. 91). Las lesiones imprudentes, por su parte, tienen asignadas una pena de un mes a dos años (art. 94) ${ }^{13}$. El problema estriba en que, según el art. 94, a las lesiones leves imprudentes podría caberle una pena de hasta dos años de prisión (al momento de escribir este trabajo tienen un máximo de tres años de prisión), situación que generaría una incoherencia dado que la pena máxima de las lesiones leves dolosas es de un año de prisión. Las normas involucradas pueden formalizarse del siguiente $\operatorname{modo}^{14}$ :

Normas formuladas:

N89: LVS \& DOL $\Rightarrow$ OS $(1 \mathrm{~m} / 1 \mathrm{a})$

N90: GRV \& DOL $\Rightarrow$ OS $(1 \mathrm{a} / 6 \mathrm{a})$

N91: GVM \& DOL $\Rightarrow$ OS (3a/10a)

N94: (LVS v GRV v GVM) \& CULP $\Rightarrow$ OS $(1 \mathrm{~m} / 3 \mathrm{a})$

Normas derivadas (de N94) ${ }^{15}$ :

N94(1): LVS \& CULP $\Rightarrow$ OS (1m/3a) (por eliminación de la disyunción en N94)

N94(2): GRV \& CULP $\Rightarrow$ OS (1m/3a) (por eliminación de la disyunción en N94)

N94(3): GVM \& CULP $\Rightarrow$ OS (1m/3a) (por eliminación de la disyunción en N94)

SANCINETTI configura la existencia del problema de las penas entre las normas N89 y N94(1) a partir del principio de proporcionalidad según el cual «toda reacción del derecho criminal — sea pena o medida de seguridad — debe guardar relación con la gravedad del hecho y los fines de la pena» ${ }^{16}$. Según el autor, la proporcionalidad supone que «toda regulación del Estado debe ser razonable, por tanto, regida por una correcta ponderación de las relaciones entre la injerencia del Estado, los fines a alcanzar con la reacción y los derechos afectados por ella» ${ }^{17}$.

SANCINETTI señala que el principio de proporcionalidad no está previsto en el texto constitucional, por lo que cabe construirlo mediatamente, proponiendo su cons-

12 SAnCinetTi, Casos de Derecho Penal, Parte General, t. I, 3. . ed., Buenos Aires, Hammurabi, 2005, 78 y ss.

13 Con posterioridad al análisis de SANCINETTI en «Casos...» se modificó la escala penal de las lesiones imprudentes del art. 94, las cuales ahora ostentan un máximo de tres años de prisión (Ley 25.189, BO de 28 de octubre de 1999).

${ }_{14}$ Este modo de formalización lo he sostenido en ALONSO, Interpretación de las normas y derecho penal, Buenos Aires, Editores del Puerto, 2006, 142 y ss.

${ }^{15}$ Las normas derivadas por el criterio de deducibilidad pertenecen al sistema normativo (CARACCIOLO, Sistemas Jurídicos. Problemas Actuales, Madrid, Centro de Estudios Constitucionales, 1988, 61 y ss.).

16 SANCINETTI, op. cit., 2005, 79.

17 Ibid. 
trucción a partir del principio de igualdad (art. 16 Constitución Argentina) y a partir del mandato constitucional de «regulación razonable» (art. 28).

Con relación a la pena prevista para las lesiones leves dolosas (art. 89, de un mes a un año) y la prevista para las lesiones culposas, dentro de las que se encuentran las leves culposas (art. 94, de un mes a dos/tres años), el autor señala que:

todo hecho doloso implica también un comportamiento descuidado (lo más descuidado es elevar el riesgo todo lo posible ex profeso) [...] el texto legal, tal como surge a primera vista, viola el principio de proporcionalidad, puesto que una acción descuidada que pone en peligro un bien jurídico y que efectivamente termina dañándolo no puede estar en peor situación que si el autor hubiera actuado adrede con el mismo resultado. Dicho brevemente: en principio, el hecho doloso es más grave, ceteris paribus, que un hecho imprudente ${ }^{18}$.

La propuesta de SANCINETTI es un ejemplo claro de obtención de un principio implícito por derivación de principios explícitos más generales y puede graficarse del siguiente modo:

Constitución

Principios explícitos

Principio implícito más general

Principio implícito más específico
Art. 16

Principio de igualdad

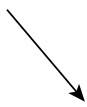

Principio de proporcionalidad

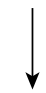

Los delitos dolosos deben estar penados más severamente que los delitos imprudentes, ceteris paribus

DOL > IMP

En cuanto al ajuste necesario para tornar coherente el sistema incoherente (ajuste eventualmente a cargo de un tribunal), SANCINETTI plantea dos alternativas: a) entender que las lesiones imprudentes leves han devenido impunes, o $b$ ) entender que las lesiones leves dolosas pueden ser penadas hasta con dos/tres años de prisión, que es la misma pena que la de las lesiones dolosas imprudentes.

Queda una tercera alternativa, que SANCINETTI no explora: $c$ ) entender que a las lesiones leves culposas le corresponde la misma pena que la de las lesiones leves dolosas, esto es, de un mes a un año de prisión. Dejaré para más adelante cuál de las tres alternativas configura, a mi juicio, el mejor ajuste coherentista.

\section{2. ¿Qué tipo de derivación es la presentada por SANCINETTI?}

La derivación propuesta por SANCINETTI es del primer tipo: a partir de principios explícitos muy generales se construye un principio implícito menos general.

18 SANCINETTI, op. cit., 2005, 81. 
No se trata de una derivación lógica ya que de los principios explícitos constitucionales invocados no se llega al principio DOL > IMP por medio de alguna regla lógica de inferencia (modus ponens, ley del refuerzo del antecedente, por ejemplo).

A mi juicio, la mejor explicación metodológica para esta forma de obtención de principios implícitos es el modelo de la coherencia analizado por autores como Neil MACCORMICK y Amalia AMAYA, según el cual la relación entre lo muy general y lo menos general no es lógica sino argumentativa o de ajuste.

Un ejemplo de este tipo de subsunción coherentista es el siguiente:

Estrato de principio explícito general $\mathrm{P}^{19}$ :

P1: Los imputados en causa penal tienen derecho a no declarar contra sí mismos.

Estrato de normas menos generales $\mathrm{NA}^{20}$ :

NA1: El imputado en causa penal no tiene obligación de declarar.

NA2: Si el imputado en causa penal desea declarar, no lo hará bajo juramento de decir verdad ni bajo pena de falso testimonio.

Estrato de normas menos generales $\mathrm{NB}^{21}$ :

NB1: El imputado en causa penal no tiene obligación de declarar.

NB2: Si el imputado en causa penal desea declarar, lo hará bajo juramento de decir verdad y bajo pena de falso testimonio.

Estrato de normas menos generales $\mathrm{NC}^{22}$ :

NC1: El imputado en causa penal tiene obligación de declarar.

NC2: El imputado en causa penal declara bajo juramento de decir verdad y bajo pena de falso testimonio.

Se advierte que los estratos de normas NA y NB son coherentes con el principio explícito P1. El estrato de normas NC, por su parte, es incoherente con el principio explícito P1. También se advierte que, más allá de que los estratos normativos NA y NB sean coherentes con el principio P1, NA y NB entre sí son inconsistentes: puntualmente la norma NA2 es contradictoria lógicamente con la norma NB2. Ello explica, al menos parcialmente, que la relación de coherencia de MACCORMICK no es lógica: P1 no implica lógicamente a los estratos NA y NB, sino que simplemente los «soporta» (o «se ajustan a»); complementariamente P1 no solo no implica sino que tampoco «no soporta» al estrato de normas NC.

19 Esta redacción es una versión simplificada de la garantía contenida en el art. 18 de la Constitución Argentina: «Nadie puede ser obligado a declarar contra sí mismo».

20 Estas normas son una versión simplificada de los arts. 296 y ss. del Código Procesal Penal de la Nación Argentina.

${ }^{21}$ Estas normas son una versión simplificada de las normas procesales penales denominadas «Advertencias Miranda», pronunciadas por la Corte Suprema de Estados Unidos. Un análisis de las mismas lo encontramos en el trabajo de MONTENEGRO, «Todo lo que (no) diga puede ser usado en su contra: Hacia una concepción más honesta de la valoración del silencio y otras derivaciones de la garantía contra la auto-incriminación en el proceso penal comparado», en AlONSO (comp.), Racionalidad en el Derecho, Buenos Aires, Eudeba, 2016, en prensa.

22 Estas normas presentan cierta analogía con las exigencias procesales que eran impuestas a los acusados de herejía en la inquisición. Sobre este punto puede verse el trabajo de TEDESCO, «El ritual de la confesión», El acusado en el ritual judicial. Ficción e imagen cultural, Editorial del Puerto, 2007. 
Volviendo al ejemplo de SANCINETTI el principio que el autor propone («los delitos dolosos deben estar penados más severamente que los delitos imprudentes, ceteris paribus - DOL > IMP-) es un principio «soportado» por los principios constitucionales explícitos que el autor invoca (igualdad y razonabilidad), pero tal relación es débil en el sentido de que podría haber otros principios implícitos menos generales alternativos «soportados» por los principios constitucionales enumerados (por ejemplo el principio «ceteris paribus los delitos imprudentes no deben estar penados más severamente que los delitos dolosos» - IMP $\leq$ DOL-), como también supone que podría haber otros principios mas generales explícitos que «soporten» el principio implícito (por ejemplo, el principio constitucional de culpabilidad podría utilizarse como soporte del principio propuesto por SANCINETTI —DOL > IMP — como el que sugerí anteriormente IMP $\leq$ DOL).

\section{LOS PRINCIPIOS IMPLÍCITOS COMO GENERALIZACIÓN A PARTIR DE NORMAS POSITIVAS MÁS ESPECÍFICAS}

Siguiendo con el ejemplo analizado por SANCINETTI, analizaré la posibilidad de justificar la obtención del principio implícito «los delitos dolosos deben estar penados más severamente que los delitos imprudentes, ceteris paribus» (DOL > IMP) por la vía de la generalización, presentada como vía $b$ ) del punto 3 del presente.

La vía de la «generalización» supone tomar como punto de partida aquellas normas penales, más precisamente aquellos pares de normas penales, que regulan, ceteris paribus, delitos dolosos y delitos imprudentes. Los pares de normas en cuestión son los siguientes:

N79: HMC \& DOL $\Rightarrow$ OS $(8 \mathrm{a} / 25 \mathrm{a})$
N84: HMC \& IMP $\Rightarrow$ OS $(6 \mathrm{~m} / 5 \mathrm{a})$
N85: ABT \& DOL $\Rightarrow$ OS $(3 \mathrm{a} / 10 \mathrm{a})$
N87: ABT \& IMP $\Rightarrow$ OS $(6 \mathrm{~m} / 2 \mathrm{a})$
N89: LVS \& DOL $\Rightarrow$ OS $(1 \mathrm{~m} / 1 \mathrm{a})$
N94 (1): LVS \& IMP $\Rightarrow$ OS $(1 \mathrm{~m} / 3 \mathrm{a})$

[(por simplificación de la disyunción en N94: (LVS v GRV v GVM) \& IMP $\Rightarrow$ OS $(1 \mathrm{~m} / 3 \mathrm{a})]$

N90: GRV \& DOL $\Rightarrow$ OS $(1 \mathrm{a} / 6 \mathrm{a})$

N94 (2): GRV \& IMP $\Rightarrow$ OS $(1 \mathrm{~m} / 3 \mathrm{a})$

[(por simplificación de la disyunción en N94:(LVS v GRV v GVM) \& IMP $\Rightarrow$ OS $(1 \mathrm{~m} / 3 \mathrm{a})]$

N91: GVM \& DOL $\Rightarrow$ OS (3a/10a)

N94 (3): GVM \& IMP $\Rightarrow$ OS (1m/3a)

[(por simplificación de la disyunción en N94:(LVS v GRV v GVM) \& IMP $\Rightarrow$ OS $(1 \mathrm{~m} / 3 \mathrm{a})]$

N176: QBR \& DOL $\Rightarrow$ OS $(2 \mathrm{a} / 6 \mathrm{a})$

N177: QBR \& IMP $\Rightarrow$ OS $(1 \mathrm{~m} / 1 \mathrm{a})$

N186 (1): INC \& DOL $\Rightarrow$ OS (3a/10a)

$\mathrm{N} 189$ (1): INC \& IMP $\Rightarrow$ OS $(1 \mathrm{~m} / 1 \mathrm{a})$ 


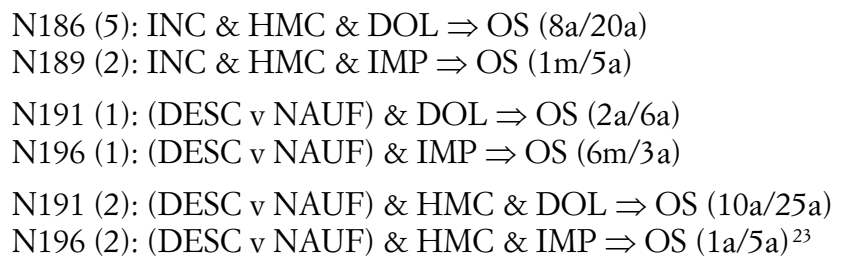

Se trata de diez pares de normas; en nueve de ellos se verifica que, ceteris paribus, los delitos dolosos están más severamente penados que los delitos imprudentes. En único par que no respeta el principio en cuestión es el analizado por SANCINETTI (el tercer par de normas, N89 vs. N94(1). Un gráfico representativo de esta generalización podría ser el siguiente:

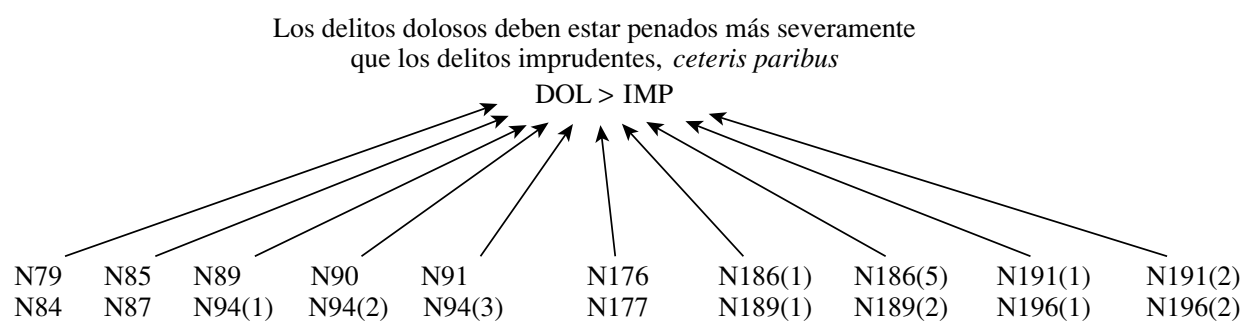

En esta vía $b$ ), la obtención de principios implícitos por generalización a partir de normas más específicas se podría justificar por varias metodologías que tienen ciertos puntos en común.

\subsection{La «inducción completa» de ALCHOURRón y BULYGIN}

En Sistemas Normativos, AlCHOURRÓN y BuLYGIN analizan la llamada «inducción jurídica» tomando como ejemplo las siguientes cuatro normas ${ }^{24}$ :

$$
\begin{aligned}
& \text { N1: } \neg \text { BFE\&BFA\&TO } \Rightarrow \text { OR } \\
& \text { N2: } \neg \text { BFE \& } \neg \text { BFA \&TO } \Rightarrow \text { OR } \\
& \text { N3: } \neg \text { BFE\&BFA\& } \neg \mathrm{TO} \Rightarrow \text { OR } \\
& \text { N4: } \neg \text { BFE\& } \neg \text { BFA } \neg \neg \mathrm{TO} \Rightarrow \text { OR }
\end{aligned}
$$

Afirman los autores que a partir de estas cuatro normas elementales puede inducirse, completamente, la siguiente norma compleja:

$$
\mathrm{N} 5: \neg \mathrm{BFE} \Rightarrow \mathrm{OR}
$$

ALCHOURRÓN y BULYGin señalan que este tipo de inducción completa había sido analizada por ARISTÓTELES en Tópica (aunque no bajo el nombre de «induc-

23 Debe aclararse que solo se consideraron la totalidad de los pares de normas que regulan delitos dolosos y delitos imprudentes ceteris paribus del Código Penal argentino, omitiéndose considerar pares de normas de características análogas de las legislaciones especiales que establecen tipos penales por fuera del Código Penal argentino [p. ej., la Ley penal tributaria (24.769) prevé algunas formas de ilícitos imprudentes].

24 Alchourron y Bulygin. Sistemas Normativos, Buenos Aires, Astrea, 2013, 118 y ss. 
ción») como una inferencia deductiva válida, no asimilable a un silogismo; se trata de una generalización a partir de todos los casos de un género. La validez de este razonamiento depende de la posibilidad de inspeccionar todos los casos. La inducción completa requiere, por tanto, la satisfacción de la tesis de la «finitud lógica del sistema» ${ }^{25}$, tesis que determina que todos los casos posibles deben ser examinables y verificados ${ }^{26}$.

En el procedimiento de «inducción completa» analizado por ALCHOURRÓN y BULYGIN el principio es representado bajo la estructura de las reglas «caso/solución», y la relación principio/reglas es de doble (o mutua) implicación lógica: el principio implica a las reglas y la conjunción de las reglas implica al principio.

Este tipo de inducción completa solo sirve para justificar principios de sistemas normativos no patológicos, esto es sistemas normativos completos, consistentes y coherentes. Cuando un sistema normativo tiene una laguna (uno de los casos genéricos carece de solución), no es posible llegar a un principio vía inducción completa dado que uno de sus casos no es examinable. Lo mismo sucede con las antinomias. Y también sucede lo mismo con las incoherencias del tipo de la aquí analizada ${ }^{27}$; el principio «DOL > IMP» no puede ser obtenido completamente dado el mismo no es verificable en el par de casos «LVS\&DOL»y «LVS\&IMP» [normas N89 y N94(1)].

\subsection{La inducción en general}

Generalmente la referencia a la «inducción a secas» no lo es al tipo de inducción completa de ALCHOURRÓN y BULYGIN sino al de la inducción incompleta, procedimiento según el cual se obtiene o justifica una generalización a partir de un conjunto finito de casos verificados con la pretensión de que la generalización obtenida se aplique a muchos otros más casos (que los verificados). «Hay inducción cuando generalizamos a partir de un número de casos de los que algo es verdad, e inferimos que la misma cosa es verdad de una clase entera» ${ }^{28}$. Este tipo de inducción (incompleta) no es un razonamiento lógico stricto sensu, dado que la conclusión dice mucho más que de lo que se deriva de las premisas.

Dentro de este procedimiento hay un puñado de metodologías que poseen elementos en común pero también poseen sutiles diferencias: el falsacionismo, la inducción probabilística y la abducción (o inferencia a la mejor explicación —IME—) serán mi objeto de análisis en los próximos párrafos.

25 SOLER, La Interpretación de la Ley, Barcelona, Ariel, 1962, 178.

26 AlCHOURRÓn y BuLYGin, Sistemas Normativos, 2013, 126 y ss.

27 SHAUER llamaría a esta incoherencia un «caso recalcitrante», debido a que la solución del caso de lesiones leves imprudentes es incoherente con la razón subyacente DOL > IMP. También pueden verse problemas por desproporcionalidad en las penas en Alonso, Interpretación de las Normas y Derecho Penal, Buenos Aires, Editores del Puerto, 2006, punto 19.2, «tipos de incoherencias», 255 y ss.

${ }^{28}$ PIERCE, «Deducción, inducción e hipótesis», en Collected Papers, 1878. Traducción al castellano de Juan Martín Ruiz-Werner, 1970, 3. 


\subsubsection{El falsacionismo}

La más famosa explicación falsacionista del conocimiento científico fue la articulada por Karl POPPER ${ }^{29}$. Según el autor inglés, una generalización debe considerarse verdadera cuántas más veces ha sido validada en casos individuales y mientras tanto no acaezca un caso individual que falsifique la generalización. Toda verdad científica es, en tal sentido, provisoria en tanto no sea falsificada; una vez producida la falsificación la generalización ha de descartarse como enunciado científico debiendo articularse nuevas explicaciones (generalizaciones) de los casos individuales sujetos a análisis ${ }^{30}$. La falsificación de la hipótesis generalista adquiere formato de modus tollens, según el cual es falsa la hipótesis en la medida en que se ha probado que existe un caso individual que no la abastece ${ }^{31}$.

Si asumimos al falsacionismo como forma de obtención de principios jurídicos implícitos deberíamos concluir que es falso que los delitos dolosos están penados más que los imprudentes (ceteris paribus), dado que el par de normas N84 - N94(1) falsifica la generalización, del mismo modo que el lazarillo educado de SCHAUER falsifica la generalización de que los perros son molestos y poco higiénicos.

Este breve análisis demuestra que más allá de la importancia del falsacionismo en el ámbito de las ciencias naturales lo cierto es que es inadecuado para justificar generalizaciones normativas, en especial porque es insensible a la detección de experiencias recalcitrantes.

\subsubsection{La inducción probabilística}

La inducción probabilística ofrece un criterio de justificación cuantitativo. En términos simples puede señalarse que, según este criterio, una generalización obtenida mediante inducción se fundamenta en su mayor grado probabilístico respecto de otras generalizaciones rivales ${ }^{32}$.

Esta justificación cuantitativa-probabilística ha sido objeto de críticas por parte de POPPER, en punto a que en ciencias naturales todas las generalizaciones tienen el mismo grado probabilístico: cero. En efecto, si el grado probabilístico es el cociente entre los casos verificados y los casos a los que se pretende aplicar la generalización, entonces tendremos siempre un número finito $n$ de casos verificados (las observaciones efectuadas en finita cantidad de metales o finita cantidad de seres humanos) y un número infinito $\infty$ de casos a los que se pretende aplicar la generalización (todos los metales, todos los seres humanos) $(n \div \infty=0)$.

29 POPPER, La lógica de la investigación científica, Madrid, Tecnos, 1967.

30 Ibid., 78.

31 Ferrajoli, Derecho y Razón, Madrid, Trotta, 1995, 143 y ss. El autor italiano utiliza el modelo falsacionista para explicar la interacción entre confirmación y refutación (vía modus tollens) de una hipótesis fáctica acusatoria en el marco de un proceso penal.

32 Estas tesis suelen atribuirse a BACON, 1620, Novum Organum, Barcelona, 1979 (traducción al castellano de C. LITRÁN). 
Sin embargo, las críticas de POPPER al inductivismo probabilístico no son trasladables sin más al ámbito del derecho positivo, donde rige la tesis de la «finitud lógica del sistema» ${ }^{33}$.

En efecto, en el ejemplo que aquí se analiza, el principio «Los delitos dolosos están/deben estar penados más severamente que los delitos imprudentes, ceteris paribus» ostenta un grado probabilístico de 90 por 100, dado que se verifica en nueve de los diez pares de normas analizados. No se verifica en el par de normas N89 - N94(1), que es, justamente, el caso recalcitrante.

Sin embargo, la generalización probabilística en cuestión podría ser reformulada de tal modo que incluya como parte de la generalización al caso recalcitrante; por ejemplo, mediante el siguiente principio: «Los delitos dolosos deben estar penados más severamente que los delitos imprudentes, salvo en el caso de las lesiones leves». Esta nueva generalización ostenta un grado probabilístico del 100 por 100; si el único criterio de corrección de una generalización es el mayor grado probabilístico, entonces este principio es vencedor con relación al originalmente formulado ${ }^{34}$.

De esta forma, y al igual que el falsacionismo, la inducción probabilística es inadecuada para las generalizaciones normativas por ser insensible al acaecimiento de casos recalcitrantes.

\subsubsection{La abducción o inferencia a la mejor explicación (IME)}

PIERCE ${ }^{35}$ intentó marcar sutiles diferencias metodológicas entre la inducción (como generalización) y la abducción (como una búsqueda para refrendar una hipótesis):

Hay inducción cuando generalizamos a partir de un número de casos de los que algo es verdad, e inferimos que la misma cosa es verdad de una clase entera. $\mathrm{O}$, cuando hallamos que cierta cosa es verdadera de cierta proporción de casos, e inferimos que es verdadera de la misma proporción de la clase entera. La hipótesis (abducción) se da cuando encontramos alguna circunstancia muy curiosa, que se explicaría por la suposición de que fuera un caso de cierta regla general, y en consecuencia adoptamos esa suposición [...]. Se han descubierto fósiles; digamos, restos como de peces, pero muy en el interior del país. Para explicar el fenómeno, suponemos que el mar cubrió en tiempos remotos esa tierra. Esa es una hipótesis. Mientras la inducción es un razonamiento de los particulares a la ley general, la abducción es del efecto a la causa ${ }^{36}$. «La abducción es una forma de razonamiento cuya conclusión es una hipótesis» (PEIRCE, 1902).

En términos lógicos la abducción es una falacia, la de la afirmación del consecuente. Dada la estructura de un condicional ordinario, «Si A entonces B», siendo A

33 SOLER, op. cit., 1962, 178. «La tesis de finitismo es verdadera para el derecho positivo. Si el derecho positivo es el derecho creado por los hombres, entonces el número de los enunciados válidos no puede ser infinito, ya que estos han sido formulados mediante pasos sucesivos y finitos» (ALCHOURRÓN y BuLYGIN, op. cit., 1971, 132-133).

34 Para una situación similar puede verse Alonso, «Principios jurídicos implícitos y coherencia», Doxa, 36, Alicante, 2013, 373.

35 PIERCE, 1878, op. cit.

36 Ibid., 8. 
la causa hipotética y B el efecto, se afirma que A es verdadera porque B se presenta reiteradamente y $\mathrm{A}$ da una razón a la reiterada presencia de $\mathrm{B}$.

A entonces B

$\mathrm{B}$

A

A: Causa o hipótesis: se supone el hecho de que el legislador castigaría mas severamente hechos dolosos que imprudentes si los mismos son equivalentes en sus restantes condiciones relevantes.

B: Consecuente: de hecho, hay muchos pares de casos en los que ceteris paribus los hechos dolosos están más penados que los imprudentes.

C: Verificamos por abducción el antecedente A, y consideramos que A es la causa de la consecuencia B.

En términos normativos la abducción presupone la asunción de una hipótesis normativa no explicitada que nos permite dar cuenta de ciertos conjuntos de reglas explicitados. En el ejemplo la hipótesis $\alpha$ señala «los hechos dolosos están penados más severamente que los imprudentes, ceteris paribus». Dicha hipótesis $\alpha$ sería la causa de numerosos efectos; los efectos son aquellos pares de normas explicitadas en los que, de hecho, ceteris paribus, los hechos dolosos están penados más severamente que los imprudentes. Cuanta más cantidad de pares de normas explicitadas encontremos más alta será la validación de la hipótesis.

Mientras que la inducción probabilística ofrece un criterio de justificación cuantitativo, la abducción ofrece uno cualitativo: la hipótesis es la razón del efecto, su explicación. Por ello, a diferencia del falsacionismo y de la inducción probabilística, la abducción normativa es un criterio que posee resistencia a los casos recalcitrantes ya que los mismos, en la medida en que sean casos aislados, no falsifican la hipótesis ni exigen su inmediata revisión; más bien lo contrario: una vez justificada abductivamente una generalización a través de ella puede corregirse el caso recalcitrante tornándolo coherente.

\subsection{La abducción en la ciencia jurídica}

En la última década muchos jusfilósofos han destacado la fecundidad de la abducción (o inferencia a la mejor explicación) como forma de generalización en los ámbitos científico, epistemológico, normativo en general y jurídico en especial ${ }^{37}$.

Amalia AMAYA ${ }^{38}$ considera que el razonamiento abductivo es el núcleo metodológico de una concepción coherentista del derecho, distinguiendo tres etapas del mismo: a) la generación o establecimiento de hipótesis normativas que permitan explicar el material positivo preexistente (normas, precedentes judiciales); b) el refinamiento o

37 LIPTON, Inference to de Best Explanation, London, Routledge, 2004; AMAYA, «Inference to the Best Legal Explanation», en Legal Evidence and Proof: Statitics, Stories, Logic, Ashgate, Alcershot, 2009; LEITER, 2007, Naturalizing Jurisprudence, Madrid, Marcial Pons, 2012 (traducción al castellano de RATTI); LAUDAN, El estándar de prueba y las garantías en el proceso penal, Buenos Aires, Hammurabi, 2011 (traducción al castellano de Calvo Soler); TuzeT, Dover decidere, Roma, Carocci Editore, 2010.

38 AmaYA, «Diez tesis acerca de la coherencia del derecho», en La coherencia en el Derecho, Discusiones, núm. 10, Bahía Blanca (Argentina), Editorial de la Universidad Nacional del Sur, 2011, 21-64. 
elección de la mejor hipótesis de entre las plausibles, y c) la selección definitiva de la mejor hipótesis por argumentos coherentistas ${ }^{39}$. Para AmAYA la coherencia del derecho vía abducción es tanto explicativa como justificativa. Es explicativa porque determina cuál es la hipótesis (o conjunto de principios implícitos, en mi denominación) que describe mejor el material jurídico preexistente; y es justificativa porque una vez obtenida la mejor hipótesis pueden ajustarse aquellos elementos incoherentes con ella. Estos ajustes pueden ser de tres tipos: contracción (eliminación del elemento incoherente), adición (incorporación de nuevos elementos) y reinterpretación (del material jurídico considerado incoherente) ${ }^{40}$.

Juliano MARANHAO ${ }^{41}$ analiza un caso de la Corte Suprema de Justicia de Brasil (Superior Tribunal Federal de Brasil), en términos parecidos al análisis abductivo que se propone en este trabajo. El material normativo involucrado es el siguiente (del Código Penal):

Art. 124: El aborto está prohibido.

Art. 128: El aborto practicado por un médico no será castigado si:

(i) No hay otro medio de salvar la vida de la madre.

(ii) El embarazo es resultado de un abuso sexual y la madre consiente el aborto ${ }^{42}$.

Según MaranhaO, ante el acaecimiento de un caso imprevisto, el del feto anencefálico que carece de posibilidad de sobrevida, se puede adoptar una actitud conservadora, prohibiendo el aborto (esta sería la derivación lógica de normas que se utilizan como punto de partida, arts. 124 y 128), o una actitud expansiva de la impunidad mediante argumentos en base a principios obtenidos abductivamente, sosteniendo que de las dos formas de impunidad — abuso sexual y peligro de vida de la madre — se puede abducir que son impunes todos aquellos casos en los que hay un sufrimiento y afectación de dignidad de la mujer embarazada, expandiendo la impunidad al caso del feto anencefálico. El método elegido por el autor para este razonamiento es la abducción o inferencia a la mejor explicación (IME).

este método de razonamiento tiene básicamente tres pasos: a) recolección de evidencias; b) formulación de hipótesis que, de ser ciertas, basados en nuestro conocimiento, explicarían aquellas evidencias, y c) comparación y selección de las hipótesis más explicativas ${ }^{43}$.

un principio jurídico (valor moral o política pública) puede ser interpretado como la mejor explicación del propósito legislativo de la regla o de un conjunto de reglas, lo que significa que el principio se deriva lógicamente de la regla aceptada por abducción. Es la aceptación de la base de reglas lo que justifica la aceptación de un principio jurídico (como un estándar jurídicamente válido) en la medida que este último provee la mejor explicación de la primera ${ }^{44}$.

Se advierte fácilmente que la propuesta presentada en este trabajo tiene muchos puntos en común con la de MARANHAO: el principio DOL > IMP se abduce de las

39 AMAYA, «Diez tesis...», op. cit., 28-29.

40 Ibid., 30-32.

41 MARANHAO, «Coherencia en el Derecho: conservadurismo y fidelidad a la base de reglas», en La coherencia en el Derecho, Discusiones, núm. 10, Bahía Blanca (Argentina), Editorial de la Universidad Nacional del Sur, 2011, 179-215.

42 MaranhaO, op. cit., 2011, 185, 187.

43 Ibid., 192.

44 Ibid. 
reglas positivas tomadas como punto de partida; y tal principio es la mejor explicación del funcionamiento de tales reglas positivas, más allá de la existencia del problemático par de casos N89/N94(1).

\section{JUSTIFICACIONES MÚLTIPLES SOBRE BASES POSITIVAS}

El principio que estamos analizando DOL > IMP se infiere por los dos métodos que toman como partida fuentes sociales del derecho (normas jurídicas positivas). Puede graficarse del siguiente modo:

Constitución

Principios explícitos

Principio implícito más general
Art. 16

Principio de igualdad

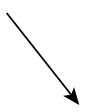

Principio de proporcionalidad
Art. 28

Principio de razonabilidad

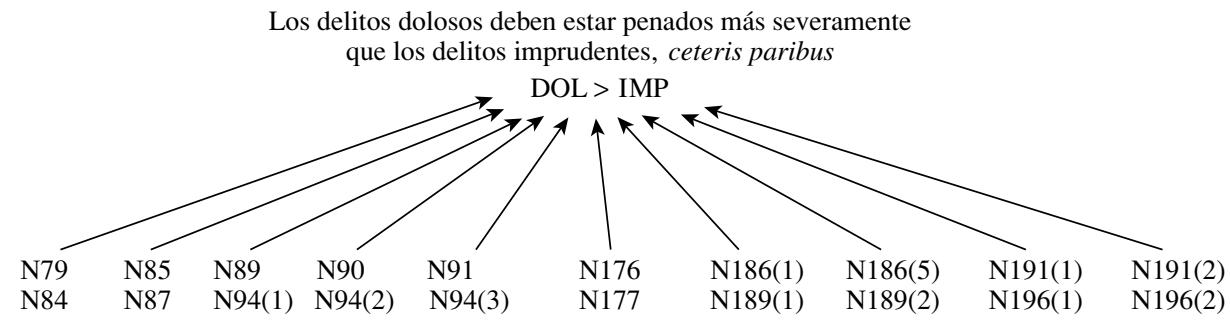

No es necesario la invocación de norma extrapositiva alguna para obtener el (o justificar al) principio DOL > IMP.

\section{INCOHERENCIAS Y AJUSTES POSIBLES}

El par de casos N89/N94(1) configura una incoherencia de acuerdo a las inferencias realizadas, tanto desde la especificación como desde la generalización abductiva. MARANHAO, considera a estas incoherencias «anormalidades»:

Los casos de anormalidad en el campo de la investigación científica son aquellos en que la evidencia contradice la teoría. Esto es, que la teoría científica ofrece una explicación cuya instanciación es inconsistente con una observación dada. En este caso, la teoría debe ser contraída para evitar la explicación equivocada, o la evidencia debe ser rechazada. Dentro del ámbito jurídico, sin embargo, si partimos de una base de reglas establecidas hay poca posibilidad de derogar reglas basadas en un caso. Si la base de reglas es consistente y no hay ninguna laguna con respecto a la acción en cuestión, entonces no debería haber tal cosa como una inconsistencia entre la «evidencia» y el conjunto de reglas aceptadas.

No obstante, podemos considerar anormalidades a aquellos casos de derrotabilidad del conjunto de reglas. Esto es, hay un caso definido por algunos factores relevantes donde 
la solución normativa derivada del conjunto de reglas establecidas es inconsistente con la solución normativa derivada del trasfondo de principios explicativo de este conjunto. SCHAUER llama a estos casos «experiencias recalcitrantes», que son el resultado de la sobre o infrainclusión de la regla. AlCHOURRÓN y BULYGIN los llaman «lagunas axiológicas» ${ }^{45}$.

En mi trabajo Interpretación de las normas y derecho penal distingo entre antinomias, lagunas normativas e incoherencias; las lagunas normativas y las antinomias son problemas internos a un sistema normativo compuesto solo de reglas, las incoherencias son un problema entre un sistema de reglas y un sistema de principios (los cuales pueden ser explícitos o implícitos); dentro de las incoherencias distingo tres tipos, el aquí analizado lo denomino contradicción axiológica, dado que el problema no estriba en el caso (porque si así fuera estaríamos ante una laguna axiológica) sino en la solución normativa ${ }^{46}$.

Volviendo al caso que analizo no queda claro el porqué y el alcance de un eventual ajuste coherentista.

A la pregunta ¿por qué ajustar la incoherencia? MACCORMICK nos responde con dos argumentos, el del universalimo y el de la inteligibilidad. El universalismo determina una exigencia de racionalidad ya que las reglas basadas en principios que las justifican son coherentes entre sí y, por ende, menos arbitrarias. La inteligibilidad permite a los sujetos normativos una comprensión de los sistemas complejos sobre la base de un conjunto de principios mas reducido que las unifican, permitiendo un mejor conocimiento del derecho y sus fines ${ }^{47}$.

ALEXY nos señala que en la hipótesis de un sistema normativo incoherente con un principio explícito (o conjunto de principios) de raigambre constitucional, parece claro que el juez puede modificar el sistema, tornándolo coherente, en virtud del criterio lex superior. En razón de la jerarquía existente, el sistema de principios constitucionales «derrota» al sistema normativo infraconstitucional ${ }^{48}$.

Pero la hipótesis analizada en este trabajo es diferente, dado que la incoherencia se presenta entre el sistema normativo y los principios implícitos de él obtenidos. Dado que no puede predicarse una relación de jerarquía entre el conjunto de normas y el conjunto de principios implícitos, la derrotabilidad de las normas en virtud de los principios implícitos no es clara.

Un camino para fundar tal derrotabilidad sería sostener que cuando un sistema normativo se subsume en un conjunto de principios implícitos que establece relaciones de orden, la violación de esas relaciones de orden es, además, una violación a la igualdad ante la ley, principio explícito de raigambre constitucional, positivizado en la mayoría de los sistemas constitucionales contemporáneos ${ }^{49}$.

${ }^{45}$ Ibid., 203-204.

46 Alonso, op. cit., 2006, 255 y ss.

47 MacCormick, Rhetoric and the Rule of Law. A Theory of Legal Reasoning, Oxford University Press, 2005, 193

${ }_{48}$ Esta es la tesis de la «Primacía Constitucional», sostenida por MORESO en La indeterminación del derecho y la interpretación de la Constitución, Madrid, Centro de Estudios Políticos y Constitucionales, 1997, 165 y ss.

49 En la Constitución de la República Argentina, el principio de igualdad ante la ley está consagrado en el art. 16. En «Principios Jurídicos Implícitos y coherencia» (ALONSO, 2013) he desarrollado en detalle los fundamentos de porqué un principio implícito puede derrotar a un conjunto de reglas explícitas. 
En nuestro caso, dado el principio implícito que exige DOL > IMP el par de normas que viola tal relación configura una incoherencia que puede ser derrotada en virtud del principio constitucional de igualdad ante la ley.

Como señala ALEXY la exigencia constitucional de igualdad no se limita a la igualdad en la aplicación del derecho sino que incluye también la igualdad en la formulación del derecho ${ }^{50}$.

En cuanto al ajuste en concreto que debería hacerse recordemos que SANCINETTI proponía dos formas de resolver la incoherencia: aumentar la pena de las lesiones dolosas leves hasta tres años, o tornar impunes las lesiones leves imprudentes.

La primera solución no es posible con el principio que propone SANCINETTI, ya que el mismo exige que lo doloso sea más penado que lo imprudente y en la propuesta de SANCINETTI la pena sería equivalente. Para sustentar esta solución el principio debería ser DOL $\geq$ IMP, «Lo doloso debe estar más o igual penado que lo imprudente ceteris paribus», principio que también es soportado por el sistema normativo que analizamos.

Esto demuestra, que tanto por instanciación como por abducción hay más de un principio soportado; pero ello, no significa que soporte cualquier principio. Por ejemplo, el principio «lo imprudente debe estar mas penado que lo doloso (IMP > DOL) », y el principio lo imprudente debe estar más o igual penado que lo doloso (IMP $\geq \mathrm{DOL}$ ), no son soportados ni por instanciación ni por abducción.

Resta una solución que SANCINETTI no explora que es determinar que las lesiones leves imprudentes tengan una pena inferior a las lesiones leves dolosas, o igual en su máximo, si es que aceptamos el principio DOL $\geq$ IMP. Si esto es así, el gráfico definitivo sería el siguiente:

Constitución

Principios explícitos

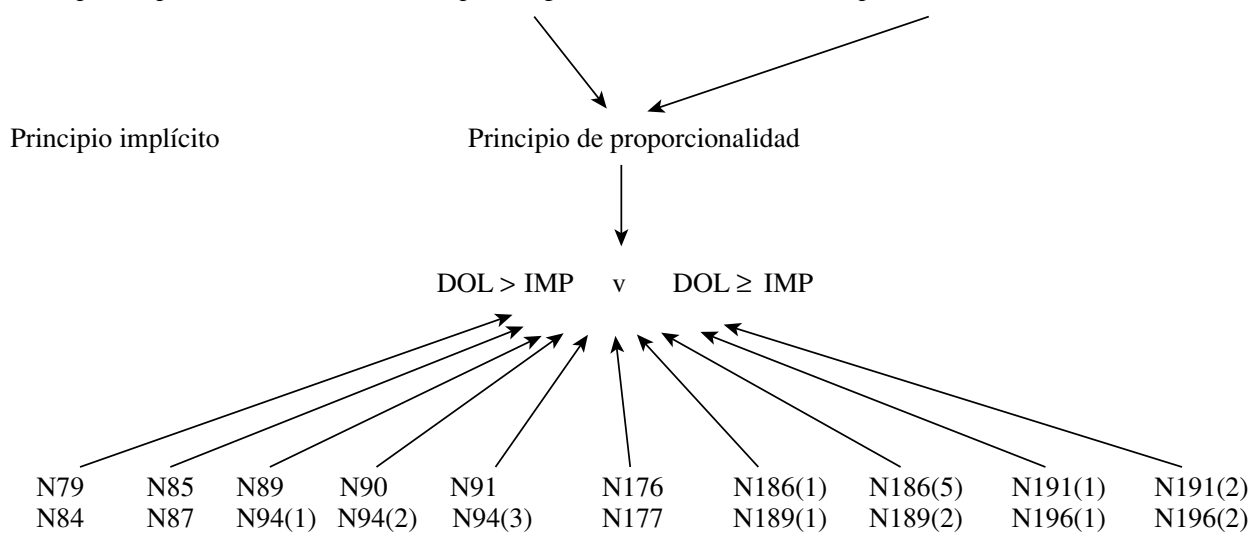

50 AleXy, La Teoría de los Derechos Fundamentales, Madrid, Centro de Estudios Constitucionales, 1993 (traducción al castellano de GARZÓN VALDÉs y ZiMMERLING), 387. 
Los tres ajustes posibles son:

NS1: LVS \& DOL $\Rightarrow$ OS $(1 \mathrm{~m} / 3 \mathrm{a})^{51}$

NS2: LVS \& IMP $\Rightarrow$ VS $^{52}$

NA1: LVS \& IMP $\Rightarrow$ OS $(1 \mathrm{~m} / 1 \mathrm{a})^{53}$

La norma NA1 es, a mi juicio, la que satisface la exigencia de MARANHAO ${ }^{54}$ de «expansión minimal», esto es, la modificación mínima necesaria para tornar coherente el sistema normativo con los principios que lo soportan y dotan de sentido, y que sacrifique la menor cantidad de elementos de la base. Esta «expansión minimal» es con relación a una eventual decisión judicial. Si se pretende coherentizar el sistema mediante una reforma legislativa la competencia reformadora es mayor; por ejemplo, el legislador podría declarar impunes las lesiones leves imprudentes.

\section{CONSIDERACIONES FINALES. UN JUSPOSITIVISMO COHERENTISTA}

Las versiones de la coherencia jurídica más difundidas son aquellas que propician versiones holistas del derecho como la de Ronald DwORKIN, a fines del siglo pasado, o la de Juan Manuel PÉREZ BERMEJO recientemente ${ }^{55}$.

Respecto de DWORKIN ya hemos dicho bastante a lo largo del trabajo, cabe agregar que también Joseph RAZ expresó su contrariedad contra estas versiones holistas de la coherencia al señalar que la teoría del derecho como integridad de DwORKIN transfiere la base de la coherencia desde el concreto conjunto de reglas a un conjunto de principios articulados de manera abstracta vinculado con las evaluaciones morales del intérprete. El resultado es que los principios ganan un estado prioritario en lugar de cumplir un rol explicativo secundario ${ }^{56}$.

PÉREZ BERMEJO, por su parte, sostiene que la evolución de los modelos jurídicos discurre desde el fundacionalismo al coherentismo. El fundacionalismo ostenta una continuidad estructural entre el jusnaturalismo y el juspositivismo del siglo XX, dado que ambos defienden que el derecho es un sistema jerárquico de tipo piramidal o de estratos, axiomático y cerrado, que apela al método de la lógica deductiva como único método relevante, y donde los jueces tienen un rol pasivo, limitado al silogismo deóntico, esto es, la aplicación de la norma general al caso individual ${ }^{57}$. Esta estructura fundacionalista, según PÉREZ BERMEJO, se presenta en autores jusnaturalistas como LEIBNIZ y juspostivistas como KELSEN, BOBBIO, AlCHOURRÓn y Bulygin. Lo común

51 NS1 es la norma 1 de SANCINETI, se eleva la pena original de las leves dolosas ( $1 \mathrm{~m} / 1 \mathrm{a})$ hasta el máximo de las imprudentes $(1 \mathrm{~m} / 3 \mathrm{a})$.

52 NS2 es la norma 2 de SANCINETTI que declara la impunidad de las lesiones leves imprudentes (VS: prohibido sancionar). $(1 \mathrm{~m} / 1 \mathrm{a})$

53 NA1 es la norma que propone reducir la pena de las leves imprudentes $(1 \mathrm{~m} / 3 \mathrm{a})$ a la de las leves dolosas

${ }^{54}$ MaranhaO, op. cit., 197.

55 Pérez Bermejo, Coherencia y Sistema Jurídico, Madrid, Marcial Pons, 2006.

$56 \mathrm{RAz}$, «The value of coherence», en Ethics in the Public Domain, Oxford University Press, 1994.

57 PÉREZ Bermejo, op. cit., 2006, 29 y ss. 
en todas estas teorías, entre otras características, es que no pueden articular argumentos coherentistas, ni siquiera como los sostenidos en este trabajo.

Para el coherentismo de PÉREZ BERMEJO un sistema jurídico está compuesto principalmente por principios ideológicos que no se infieren de las normas jurídicas; la validez de estos principios depende de sentimientos de conveniencia, justicia y moral y no de criterios tipo origen o pedigree; a diferencia de las reglas estos principios poseen dimensión de peso, por lo cual su papel en el razonamiento jurídico es diferente al papel de las reglas. Las reglas positivas no sirven para ordenar al material jurídico ya que el carácter sistemático del derecho reside en los principios. Un derecho sin principios sería un grupo confuso de disposiciones asistemáticas.

Finalmente, para PÉREZ BERMEJO, cada caso de aplicación del derecho «convoca diversos principios igualmente válidos, exige un examen del peso de importancia de los mismos e implica una ponderación juiciosa de desempate» ${ }^{58}$; «describir, interpretar o aplicar una norma jurídica significa dar cuenta de todo el derecho de una comunidad ${ }^{59}$.

Comparto las críticas de Giovanni RATTI en punto a que «parece dudoso que haya juristas capaces "o interesados en" conocer y aplicar todo el orden jurídico de una comunidad, compuesto por un sinnúmero de disposiciones jurídica, a un caso concreto» ${ }^{60}$.

TARELLO ya había advertido que generalmente los principios utilizados por los juristas y los dogmáticos para sistematizar el derecho suelen ser relativos a subsistemas legislativos, y no tan generales como los principios defendidos por los coherentistas (TARELLO hace referencia al principio de conservación del contratos; al principio favor debitoris, al principio pro operario, etc. ${ }^{61}$.

Como señala MARANHAO, esta era la idea de SAVIGNY, cuando afirmaba:

la exigencia de coherencia en la descripción de todo el conjunto de normas válidas no refleja nuestra verdadera manera de entender el derecho. Las preguntas prácticas sobre cuál es el derecho son usualmente hechas para saber qué es esto que consideremos derecho válido dice sobre tal acción en estas y aquellas circunstancias. Para resolverlas solo un conjunto de reglas basado en fuentes necesita estar activo. Es innecesario e irrazonable exigir una descripción idealizada y comprehensiva de todo el conjunto de reglas válidas. Por ejemplo, ¿cuál es la relevancia de principios relacionados con reglas sobre interconexión de redes de telecomunicación respecto a la permisión y prohibición del aborto? ¿En qué sentido deberían las reglas sobre telecomunicaciones y sobre el aborto formar un todo coherente?

En definitiva, si entendemos que el juspositivismo se limita a las dos tesis que aquí se sostienen (las fuentes sociales del derecho y la separación entre derecho y moral), entonces no hay razón para argüir que el mismo no puede utilizar a la abducción como mecanismo para validar principios jurídicos implícitos por generalización; tampoco podría argüirse que desde el juspositivismo no podría articularse un modelo de cohe-

${ }^{58}$ Ibid., 126.

59 Ibid., 135.

60 RATTI, «La coherentización de los sistemas jurídicos», en El gobierno de las normas, Madrid, Marcial Pons, 2013, 80

${ }^{61}$ TARello, La interpretación de la Ley, Milano, Giuffrè, 1980, 384-385. En el mismo sentido RatTI, op. cit., $2013,78$. 
rencia para validar principios implícitos por instanciación. Puntualmente, es posible una versión positivista de los principios implícitos y de la coherencia, máxime cuando el análisis se acota a sistemas normativos específicos.

\section{BIBLIOGRAFÍA}

Alchourrón, C., y Bulygin, E., 1971: Normative Systems. Las citas se corresponden con la versión castellana de los autores, titulada Introducción a la Metodología de las Ciencias Jurídicas y Sociales, Buenos Aires: Astrea, 1975.

ALEXY, R., 1986: Theorie der Grundrechte. Las citas se corresponden con la traducción al castellano de E. GARZÓN VALdÉs y R. ZimmerLing, 1993, La Teoría de los Derechos Fundamentales, Madrid: Centro de Estudios Constitucionales.

Alonso, J., 2006: Interpretación de las Normas y Derecho Penal, Buenos Aires: Editores del Puerto.

— 2013: «Principios jurídicos implícitos y coherencia», Doxa, 36, Alicante.

AmayA, A., 2009: «Inference to the Best Legal Explanation», Legal Evidence and Proof: Statitics, Stories, Logic, Ashgate: Alcershot.

- 2011: «Diez tesis acerca de la coherencia del derecho», La coherencia en el Derecho, Discusiones, núm. 10, Bahía Blanca (Argentina): Editorial de la Universidad Nacional del Sur.

Atienza, M., y Ruiz Manero, J., 1996: Las piezas del derecho. Teoría de los enunciados jurídicos, Madrid: Ariel Derecho.

BACON, F., 1620: Novum Organum. Las citas se corresponden con la traducción al castellano de C. LITRÁN, Barcelona: Editorial de Bolsillo, 1979.

Bulygin, E., 1966: «Sentencia judicial y creación de derecho», en C. Alchourrón y E. BulYGIN, Análisis lógico y derecho, Madrid: Centro de Estudios Constitucionales, 1991.

Caracciolo, R., 1988: El sistema jurídico. Problemas actuales, Madrid: Centro de Estudios Constitucionales.

Dworkin, R., 1977: «The model of rules (I)», Taking Rights Seriously. Las citas se corresponden a la traducción al castellano de M. GUASTAVINO, «El modelo de las normas (I)», Los derechos en serio, Barcelona: Ariel, 1984.

FerRAJOLI, L., 1995: Derecho y Razón, Madrid: Trotta.

Guastini, R., 1990: Dalle fonti alle norme, Torino: Giappichelli.

HART, H., 1961: The Concept of Law. Las citas se correspondes con la traducción al castellano de G. CARrió, El concepto de derecho, Buenos Aires: Abeledo Perrot, 1992.

LAUDAN, L., 2011: El estándar de prueba y las garantías en el proceso penal, Buenos Aires: Hammurabi (traducción al castellano de Raúl CALVO SOLER).

LeITER, B., 2007: Naturalizing Jurisprudence, Madrid: Marcial Pons, 2012 (traducción al castellano de Giovanni RATTI).

LiPTON, P., 2004: Inference to de Best Explanation, London: Routledge.

MacCormick, N., 1978: Legal Reasoning and Legal Theory, Oxford: Oxford University Press.

- 1984: «Coherence in Legal Justification», Theory of Legal Science, Dordrecht: Reidel Publishing, 231-251.

- 2005: Rhetoric and the Rule of Law. A Theory of Legal Reasoning, Oxford: Oxford University Press.

MARAnHAO, J., 2011: «Coherencia en el Derecho: conservadurismo y fidelidad a la base de reglas», La coherencia en el Derecho, Discusiones, núm. 10, Bahía Blanca (Argentina): Editorial de la Universidad Nacional del Sur. 
Montenegro, L., 2016: «Todo lo que (no) diga puede ser usado en su contra: Hacia una concepción más honesta de la valoración del silencio y otras derivaciones de la garantía contra la auto-incriminación en el proceso penal comparado», en Juan Pablo AlONSO (comp.), Racionalidad en el Derecho, Buenos Aires: Eudeba.

MoRESO, J., 1997: La indeterminación del derecho y la interpretación de la Constitución, Madrid: Centro de Estudios Políticos y Constitucionales.

Pérez Bermejo, J., 2006: Coherencia y Sistema Jurídico, Madrid: Marcial Pons.

PIERCE, Ch., 1970: «Deducción, inducción e hipótesis», Collected Papers, 1878. Traducción al castellano de Juan MARTín RUIZ-WERnER, p. 3.

POPPER, K., 1959: The Logic of Scientific Discovery, London: Routledge.

- 1982: «Postcript to The Logic Scientific Discovery», Realism and the Aim of Science, London: Taylor \& Francis.

PRIETO SANCHÍs, L., 1992: Sobre principios y normas. Problemas del razonamiento jurídico, Madrid: Centro de Estudios Constitucionales.

RATTI, G., 2013: «La coherentización de los sistemas jurídicos», El gobierno de las normas, Madrid: Marcial Pons.

RAZ, J., 1994: «The value of coherence», Ethics in the Public Domain, Oxford: Oxford University Press.

SancinetTi, M., 2005: Casos de Derecho Penal, Parte General, t. I, 3. .a ed., Buenos Aires: Hammurabi.

Schauer, F., 1991: Playing by the Rules. A Philosophical Examination of Rule -Based Decision-Making un Law and un Life, Oxford: Oxford University Press.

SOLER, S., 1962: La Interpretación de la Ley, Barcelona: Ariel, 178.

TARELLO, G., 1980: La interpretación de la Ley, Milano: Giuffre.

TEDESCO, I., 2007: «El ritual de la confesión», El acusado en el ritual judicial. Ficción e imagen cultural, Editorial del Puerto.

TuZET, G., 2010: Dover decidere, Roma: Carocci editore.

Vigo, R., 2004: La injusticia extrema no es derecho (De Radbruch a Alexy), Buenos Aires: La Ley. Zuleta, H., 1998: Razón y Elección, México: Fontamara. 